\title{
The clinical significance of the T2-FLAIR mismatch sign in grade II and III gliomas: a population-based study
}

\author{
Alba Corell ${ }^{1,2^{*}}$ D, Sandra Ferreyra Vega ${ }^{2}$, Nickoleta Hoefling ${ }^{3,4}$, Louise Carstam ${ }^{1,2}$, Anja Smits ${ }^{2,5}$, \\ Thomas Olsson Bontell ${ }^{6,7}$, Isabella M. Björkman-Burtscher ${ }^{3,4}$, Helena Carén ${ }^{8}$ and Asgeir Store Jakola ${ }^{1,2,9}$
}

\begin{abstract}
Background: The T2-FLAIR mismatch sign is an imaging finding highly suggestive of isocitrate dehydrogenase mutated (IDH-mut) 1p19q non-codeleted (non-codel) gliomas (astrocytomas). In previous studies, it has shown excellent specificity but limited sensitivity for IDH-mut astrocytomas. Whether the mismatch sign is a marker of a clinically relevant subtype of IDH-mut astrocytomas is unknown.
\end{abstract}

Methods: We included histopathologically verified supratentorial lower-grade gliomas (LGG) WHO grade II-III retrospectively during the period 2010-2016. In the period 2017-2018, patients with suspected LGG radiologically were prospectively included, and in this cohort other diagnoses than glioma could occur. Clinical, radiological and molecular data were collected. For clinical evaluation we included all patients with IDH-mut astrocytomas. In the 2010-2016 cohort DNA methylation analysis with Infinium MethylationEPIC BeadChip (Illumina) was performed for patients with an IDH-mut astrocytoma with available tissue. We aimed to examine the association of the T2-FLAIR mismatch sign with clinical factors and outcomes. Additionally, we evaluated the diagnostic reliability of the mismatch sign and its relation to methylation profiles.

Results: Out of 215 patients with LGG, 135 had known IDH-mutation and 1p19q codeletion status. Fifty patients had an IDH-mut astrocytoma and 12 of these (24.0\%) showed a mismatch sign. The sensitivity and specificity of the mismatch sign for IDH-mut detection were 26.4 and $97.6 \%$, respectively. There were no differences between patients with an IDH-mut astrocytoma with or without mismatch sign when grouped according to T2-FLAIR mismatch sign with respect to baseline characteristics, clinical outcomes and methylation profiles. The overall interrater agreement between neuroradiologist and clinical neurosurgeons for the T2-FLAIR mismatch sign was significant when all $215 \mathrm{MRl}$ examination assessed ( $\mathrm{k}=0.77, p<$ $0.001, N=215)$.

Conclusion: The T2-FLAIR mismatch sign in patients with an IDH-mut astrocytoma is not associated with clinical presentation or outcome. It seems unlikely that the IDH-mut astrocytomas with mismatch sign represent a specific subentity. Finally, we have validated that the T2-FLAIR mismatch sign is a reliable and specific marker of IDH-mut astrocytomas.

Keywords: Astrocytoma, Oligodendroglioma, Biomarkers, Decision making, prognosis

\footnotetext{
* Correspondence: alba.corell@vgregion.se

'Department of Neurosurgery, Sahlgrenska University Hospital, Gothenburg,

Sweden

${ }^{2}$ Department of Clinical Neuroscience, Institute of Neuroscience and

Physiology, University of Gothenburg, Sahlgrenska Academy, Gothenburg,

Sweden

Full list of author information is available at the end of the article
}

(c) The Author(s). 2020 Open Access This article is licensed under a Creative Commons Attribution 4.0 International License, which permits use, sharing, adaptation, distribution and reproduction in any medium or format, as long as you give appropriate credit to the original author(s) and the source, provide a link to the Creative Commons licence, and indicate if changes were made. The images or other third party material in this article are included in the article's Creative Commons licence, unless indicated otherwise in a credit line to the material. If material is not included in the article's Creative Commons licence and your intended use is not permitted by statutory regulation or exceeds the permitted use, you will need to obtain permission directly from the copyright holder. To view a copy of this licence, visit http://creativecommons.org/licenses/by/4.0/. The Creative Commons Public Domain Dedication waiver (http://creativecommons.org/publicdomain/zero/1.0/) applies to the data made available in this article, unless otherwise stated in a credit line to the data. 


\section{Background}

Lower-grade gliomas (LGG) are intra-axial neoplasms of the brain, including WHO grade II and III astrocytomas and oligodendrogliomas according to the WHO 2016 classification and The Cancer Genome Atlas Research Network [1, 2]. These subgroups are based upon determination of mutation in the isocitrate dehydrogenase genes 1 or 2 (IDH1 and IDH2), and $1 \mathrm{p} 19 \mathrm{q}$ codeletion status [3, 4]. In addition to providing tumor classification, these markers also provide important prognostic information [1, 2, 4-6].

Biomarkers are the fundamental keystones of personalized management strategies, but the traditional biomarkers analyzed in tumor tissue come into play only after the surgical procedure. Thus, in the neurosurgical decision making, image-based biomarkers are of particular interest to identify relevant subgroups of patients. The newly described imaging feature of T2-FLAIR (fluid attenuation inversion recovery) mismatch sign has gained increased attention, since it is a widely available and simple potential imaging marker to predict $I D H$ mutated (IDH-mut) 1p19q non-codeleted (non-codel) gliomas (astrocytoma) with high specificity [7, 8]. The T2-FLAIR mismatch sign (further also referred to as mismatch sign) is characterized by a hyperintense signal on T2-weighted sequences and a hypointense signal on FLAIR sequences with a hyperintense peripheral rim, see Supplementary Figs. 1, 2 and 3 as examples.

DNA methylation analysis is in the frontline of diagnostic technology in gliomas [9-11]. The tumors showing a mismatch sign on MRI differ radiologically from gliomas without the mismatch sign with their distinct features. This raises questions regarding underlying biology. Studies so far, however, have not indicated that this radiological marker is reflected by a specific biological signature $[7,12]$.

An important question remains whether $I D H$-mut astrocytomas with or without mismatch sign reflect differences of clinical relevance, including resectability, based on the T2-weighted appearance being homogeneous and well demarcated or not. For instance, if $I D H$-mut astrocytomas with mismatch sign can be depicted by neuroimaging as more "resectable", this tool would provide important information prior to surgery, where the extent of resection is of particular importance for patients with $I D H$-mut astrocytomas [13, 14]. In addition, institutional experience from neurosurgeons over the years has led to speculations about differences in texture in some tumors, being softer or more gelatinous and perhaps easier to remove. Since size, location and proximity to critical structures are of such importance in surgical decision-making, the identification of an imaging marker pointing towards factors affecting resection, could indeed alter surgical management, especially in complicated cases [15].
We aimed to evaluate clinical parameters including extent of resection with regard to mismatch sign. In addition, we analyzed if $I D H$-mut astrocytomas with mismatch sign had similar methylation profiles compared to samples without mismatch sign. Finally, we provide interrater variability between neurosurgeons and neuroradiologist and the sensitivity and specificity of the mismatch sign.

\section{Methods}

All patients in the Västra Götaland region in Sweden with newly diagnosed primary intracranial intra-axial tumors are managed in a multidisciplinary team (MDT) with weekly conferences at the Sahlgrenska University Hospital. The neurosurgical department at the Sahlgrenska University Hospital in Gothenburg covers the population of approximately 1.7 million inhabitants.

Our cohort consists of two components; one retrospective cohort and one prospective. For patient selection see flowchart in Fig. 1. We performed a retrospective collection of clinical and radiological data between 2010 and 2016, searching operation logs and pathology database, thus covering all patients with a histopathological diagnosis of a supratentorial infiltrating WHO grade II or III glioma with magnetic resonance imaging (MRI) $[1,16]$.

In the years 2017 and 2018, we included patients prospectively based on a high suspicion of LGG grade II and III, referred either to the MDT conference or directly to the neurosurgical department. In principle, our cohort consists of lesions indicative of primary brain tumor with hyperintense signal in T2 weighted images, but with no significant contrast enhancement. In this cohort, other histopathological diagnoses may be encountered (e.g. other tumor subgroups or even non-neoplastic lesions). The rationale for "blindly" including these patients was to enable the evaluation of the mismatch sign in a group with rather similar MRI appearance, but also differential diagnoses of LGG, in contrast to most previous studies that used histopathology as selection [7, 17].

We used MRI images from all patients in the retro- and prospective cohorts to perform the interrater evaluation of the T2-FLAIR mismatch sign $(N=215)$. In further clinical evaluations, we analyzed the mismatch sign in patients with known $I D H$-mut gliomas without $1 \mathrm{p} 19 \mathrm{q}$ codeletion from both cohorts $(N=50)$. The DNA methylation was performed in patients from the retrospective cohort with $I D H$-mut non-codel gliomas $(N=29)$.

Clinical variables such as patients' age, sex, symptoms at diagnosis and Karnofsky functional status [18] were recorded. For basic radiological variables we included main lobe involved, tumor border (absent, mild/moderate or conspicuous) and eloquence [19]. We analyzed patients with $I D H$-mut astrocytomas divided into two groups, with or without mismatch sign. 


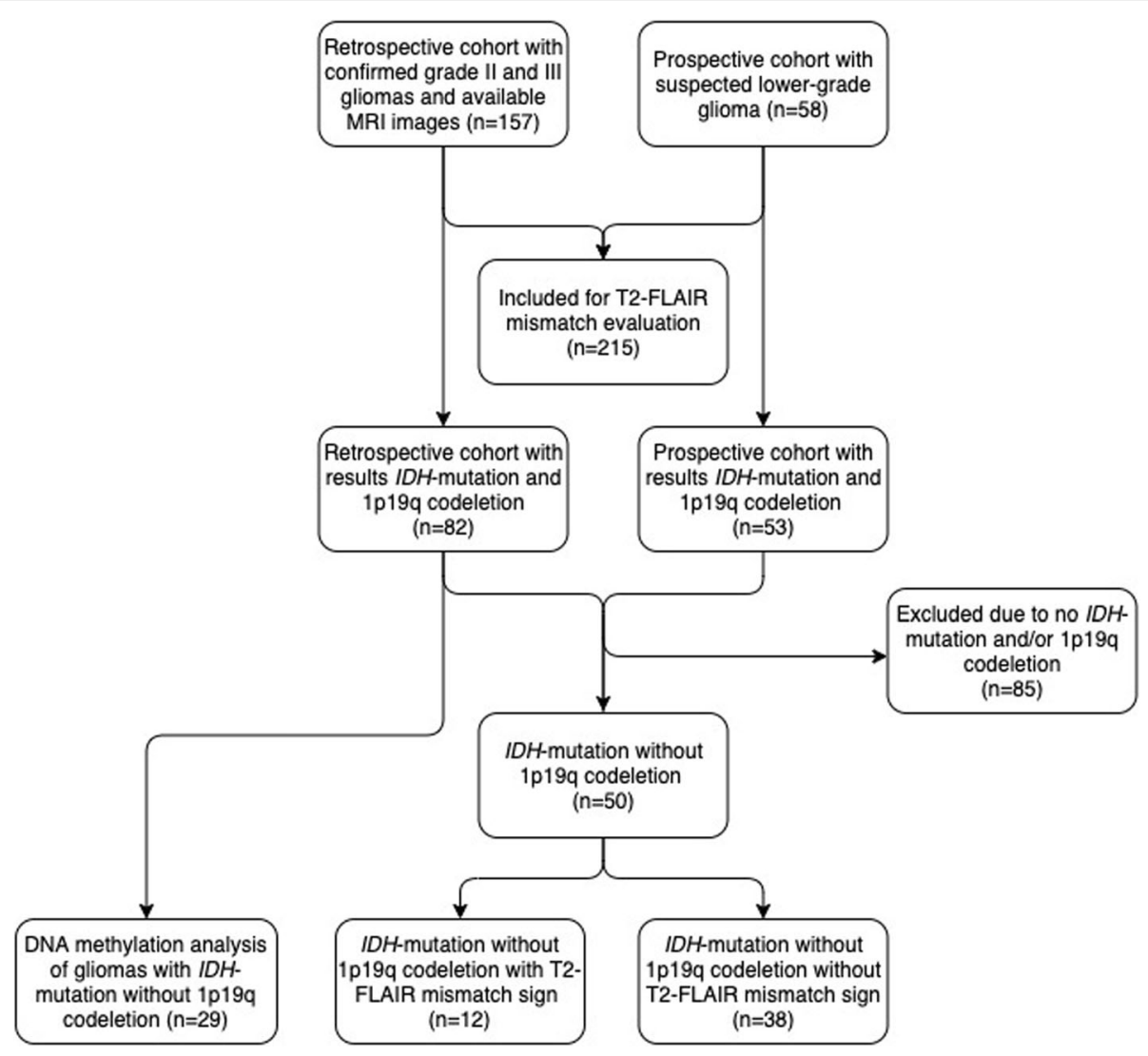

Fig. 1 Flowchart of patient inclusion

\section{Image acquisition}

MRI examinations reviewed in this project were performed at different hospitals as part of the clinical preoperative work up in these tumor patients. MRI systems used for image acquisition included both $1.5 \mathrm{~T}$ and $3.0 \mathrm{~T}$ scanners from different vendors (GE Healthcare, US; Philips, The Netherlands; Siemens Healthcare, Germany) and with different software releases. 2D sequences with accepted slice thicknesses $\leq 5 \mathrm{~mm}$ were predominant. For T2-weighted sequences, median repetition time was 4000 milliseconds (ms) and median echo time was 100 ms. For FLAIR examinations, median repetition time was $9000 \mathrm{~ms}$, median echo time was $122 \mathrm{~ms}$, and median inversion time was $2500 \mathrm{~ms}$. All scanners underwent regular maintenance by the vendors and sequences were optimized by the hospitals for clinical evaluation of brain lesions such as brain tumors.

\section{Image evaluation}

Images were analyzed for: main lobe involved (frontal, temporal, parietal, occipital, insula), side (right, left, bilateral), border (absent, mild/moderate or conspicuous), eloquence [19], T2-FLAIR mismatch (yes/no), and size (volume by tumor segmentation).

\section{T2-FLAIR mismatch analysis}

The MRI scans were evaluated for the T2-FLAIR mismatch sign as done by Patel et al [7]. Evaluation was independently performed by a neurosurgical resident (AC), a senior neurosurgeon (ASJ) and a board certified neuroradiologist $(\mathrm{NH})$. In case of disagreement between the clinicians a consensus reading was performed between the neurosurgeons and interrater agreement was calculated. The consensus was then compared to the independent reading of the neuroradiologists (interrater agreement) and in case of disagreement, a final consensus reading was performed with a senior neuroradiologist (IBB).

\section{Tumor segmentation}

Both T2 and FLAIR sequences were used for tumor volume segmentation, depending on which sequence the tumor was more clearly visible. FLAIR was often used in the preoperative MRI. Due to surgically-induced artifacts, T2 was sometimes preferred in the postoperative MRI and evaluation was done on a case-by-case basis. This selection was done up-front, and we did not segment both T2 and FLAIR sequences with later selection of the more "beneficial" one. 
The tumor volume was evaluated by semi-automatic segmentation performed with the open-source software "3DSlicer", version 4.6.2 [20]. For the segmentation of tumor volume, we used the tools "LevelTracingEffect", "WandEffect", "DrawEffect" and "PaintEffect" in the "Editor" module when appropriate. Tumor volumes were computed by the segmentation of hyperintensive areas on the T2 or FLAIR sequence on MRI examinations. Areas attributed to mainly edema without convincing signs of tumor invasion were excluded. In the gliomas with T2-FLAIR mismatch sign we used the outer margin of the peripheral rim on FLAIR images as the outer tumor border. Segmentation was performed by the neurosurgical resident (AC) with quality control in all cases from a senior neurosurgeon (ASJ) and neuroradiological expertise used in selected cases.

\section{DNA methylation array}

DNA from formalin-fixed paraffin embedded (FFPE) tumors from patients included in the retrospective cohort was isolated with the QIAamp ${ }^{\oplus}$ DNA FFPE kit (Qiagen, Hilden, Germany) according to the supplier's instructions with the addition of an extra digestion step with proteinase $\mathrm{K}$ overnight. DNA concentration was measured with the Qubit Fluorometer (Life technologies ${ }^{\mathrm{TM}}$, Carlsbad, CA, USA). Between 500 and 1000 ng DNA was bisulfite-converted with the EZ DNA methylation kit (D5001, Zymo Research, Irvine, CA, USA) and the methylation levels of restored bisulfite-converted DNA was determined with the Infinium MethylationEPIC BeadChip (Illumina ${ }^{\circ}$, San Diego, CA, USA) according to the protocols provided by the supplier.

Methylation analysis and normalization was performed as previously described [21]. Briefly, methylation data were processed with the statistical software $\mathrm{R}$ (version 3.6.1) using the Minfi [22] and ChAMP [22-24] packages. IDH mutational status was acquired using a published DNA methylation-based classifier [9]. 1p19q codeletion status was acquired through copy number variations inferred from the array. Correlation between the T2-FLAIR mismatch sign and DNA-methylation profiles was evaluated by unsupervised hierarchical clustering of the 5000 most variable CpG sites including only patients with $I D H$-mut non-codel gliomas $(N=29)$.

\section{Statistics}

All statistical analyses were done with SPSS, version 24.0 (Chicago, IL, USA). Statistical significance level was set to $p<0.05$ and all tests were two-sided. Central tendencies were presented as means \pm standard deviation (SD), or median and first quartile (Q1) to third quartile (Q3) if skewed. Categorical data were analyzed with Pearson's chi square test, but in $2 \times 2$ tables Fishers exact test was used when appropriate due to small sample. For continuous data independent sample t-test or Mann-Whitney $U$ test were used as appropriate based upon data distribution. Interrater agreement between the clinical assessment by neurosurgeons and neuroradiologist for the presence or absence of the mismatch sign was assessed with Cohen's kappa statistic (k) [25]. We considered $>0.6$ to be substantial agreement, 0.41-0.6 moderate agreement, 0.21-0.4 fair agreement and $\leq 0.2$ slight agreement [25]. To evaluate the interrater agreement, we used all patients in the retrospective and prospective cohort, regardless of molecular status. Finally, we present sensitivity and specificity for T2-FLAIR mismatch sign as a marker to identify $I D H$ mut astrocytomas.

\section{Ethics statement}

This project was approved by the regional ethical committee in the region of Västra Götaland (DNR 1067-16 and DNR 363-17).

\section{Results \\ Clinical factors and outcomes}

Our patient cohort included retro- and prospectively 135 patients with available MRI images and known status of $I D H$-mutation and $1 \mathrm{p} 19 \mathrm{q}$ codeletion. The retrospective part of the cohort included 82 patients with mean age of 45.0 years (SD 14.3) and 37 patients (45.1\%) were females. The majority of this cohort underwent resection, as opposed to biopsy only ( $N=77,93.9 \%)$.

In the prospective part, we evaluated 58 patients with a suspected LGG. This included both neoplastic and nonneoplastic diagnoses, such as limbic encephalitis. Of these, 53 patients had known status of $I D H$-mutation and $1 \mathrm{p} 19 \mathrm{q}$ codeletion. In this cohort, 22 patients were female $(41.5 \%)$ and the mean age was 47.9 years (SD 15.7). Resection was the most common surgical treatment $(n=46,86.8 \%)$. The most common histopathological diagnoses were WHO grade II or III astrocytoma $(N=27,50.9 \%)$, oligodendroglioma $(N=21,36.9 \%)$ and glioblastoma $(N=4,7.5 \%)$. Other diagnoses included non-neoplastic lesions such as limbic encephalitis. In addition, one patient (1.9\%) had other diagnosis (DNET). The mismatch sign was not present in any of the nonneoplastic diagnoses, or in the patient with DNET.

In total there were 50 patients with $I D H$-mut astrocytoma. These were grouped based upon presence of T2FLAIR mismatch sign $(N=12,24.0 \%)$ or absence $(N=38$, $76.0 \%$ ). In Table 1 we present comparison between these groups with respect to baseline characteristics, radiological variables and clinical outcome. The only significant difference was a more conspicuous tumor border in the group with mismatch sign $(p=0.02)$. There were no differences regarding tumor location, pre- and postoperative volumes, symptoms or type of surgery. Importantly, there were no differences between groups with respect to the extent of 
Table 1 Presentation and outcomes in patients diagnosed between 2010 and 2018 with IDH-mut astrocytomas ( $N=50)$, presented in relation to the T2-FLAIR mismatch sign presence or absence

\begin{tabular}{|c|c|c|c|}
\hline & Mismatch $(\boldsymbol{N}=12,24.0 \%)$ & No mismatch $(\mathrm{N}=38,76.0 \%)$ & $\boldsymbol{P}$-value \\
\hline Age, years, mean (SD) & $35.7(12.6)$ & $41.9(14.4)$ & 0.06 \\
\hline Female, n (\%) & $7(58.3)$ & $16(42.1)$ & 0.51 \\
\hline \multicolumn{4}{|l|}{ Main lobe involved, n (\%) } \\
\hline Frontal & $7(58.3)$ & $17(44.7)$ & 0.51 \\
\hline Temporal & $3(25.0)$ & $13(34.2)$ & 0.73 \\
\hline Parietal & $2(16.7)$ & $7(18.4)$ & 1.00 \\
\hline Insula & $0(0.0)$ & $1(2.6)$ & 1.00 \\
\hline \multicolumn{4}{|l|}{ Image findings, n (\%) } \\
\hline Right side & $6(50.0)$ & $15(39.5)$ & 0.74 \\
\hline Left side & $6(50.0)$ & $23(60.5)$ & 0.74 \\
\hline Bilateral/midline & $0(0.0)$ & $5(13.2)$ & 0.32 \\
\hline Conspicuous border & $9(75.0)$ & $12(31.6)$ & 0.02 \\
\hline Eloquence & $8(66.7)$ & $30(78.9)$ & 0.45 \\
\hline \multicolumn{4}{|l|}{ Symptom at diagnosis ${ }^{\mathrm{a}}, \mathrm{n}(\%)$} \\
\hline Asymptomatic & $0(0.0)$ & $3(7.9)$ & 1.00 \\
\hline Seizure & $9(75.0)$ & $28(73.7)$ & 1.00 \\
\hline ICP related & $3(25.0)$ & $11(28.9)$ & 1.00 \\
\hline Deficit(s) & $0(0.0)$ & $5(13.2)$ & 0.32 \\
\hline Language deficit & $2(16.7)$ & $2(5.3)$ & 0.24 \\
\hline Visual deficit & $2(16.7)$ & $4(10.5)$ & 0.62 \\
\hline Cognitive changes & $3(25.0)$ & $8(21.1)$ & 1.00 \\
\hline Other symptoms & $1(8.3)$ & $10(26.3)$ & 0.25 \\
\hline \multicolumn{4}{|l|}{ Type of surgery, n (\%) } \\
\hline Resection & $11(91.7)$ & $37(97.4)$ & 0.43 \\
\hline \multicolumn{4}{|l|}{ Volumetric measurements } \\
\hline Preoperative volume, ml, median (Q1-Q3) & $47.4(29.71-113.61) N=11$ & $63.3(26.52-115.32) N=37$ & 0.91 \\
\hline Postoperative volume, ml, median (Q1-Q3) & $5.9(1.67-14.81) \mathrm{N}=11$ & $5.0(0.34-16.42) \mathrm{N}=37$ & 0.99 \\
\hline Extent of resection, median \% (Q1-Q3) & $87.9(73.60-96.63) \mathrm{N}=11$ & $89.2(48.50-99.75) \mathrm{N}=37$ & 0.91 \\
\hline \multicolumn{4}{|l|}{ WHO grade, n (\%) } \\
\hline WHO grade II & $5(41.7)$ & $24(63.2)$ & 0.31 \\
\hline WHO grade III & $6(50.0)$ & $13(34.2)$ & 0.50 \\
\hline WHO grade IV & $1(8.3)$ & $1(2.6)$ & 0.43 \\
\hline
\end{tabular}

${ }^{\mathrm{a}}$ More than one symptom at presentation possible. Other symptoms included paresthesia, vertigo, dysphagia, among others

resection $(87.9 \%$ with mismatch sign and $89.2 \%$ without mismatch sign, $p=0.91$ ) or survival (median of 85 months in cohort with mismatch sign vs 65 months without mismatch sign, $\mathrm{p}=0.91$ ).

\section{Diagnostic properties}

Nine patients $(10.9 \%)$ in the retrospective part of the cohort showed mismatch signs and all of them had $I D H$-mutated gliomas. There were no patients with a glioma with $I D H$-wild type and mismatch sign in the retrospective cohort. However, we identified two patients with $1 \mathrm{p} 19 \mathrm{q}$ codeletion (codel) tumors (oligodendrogliomas) and mismatch sign (see Fig. 2a-b and 3a-b). In the prospective cohort, the lesion of 5 patients (8.6\%) showed a mismatch sign, all of them being $I D H$-mut astrocytoma whereof one being WHO grade IV (glioblastoma, see Fig. 4a-b). One patient with a DNET did not show a positive T2-FLAIR mismatch sign.

In the joint cohort with known molecular status, including both $I D H$-mutation and $1 \mathrm{p} 19 \mathrm{q}$ codeletion $(N=$ $135)$, the specificity for $I D H$-mut astrocytomas was $97.6 \%$ and the sensitivity was $26.4 \%$. The positive predictive value (PPV) was $85.7 \%$ and the negative predictive value (NPV) was $67.7 \%$. 

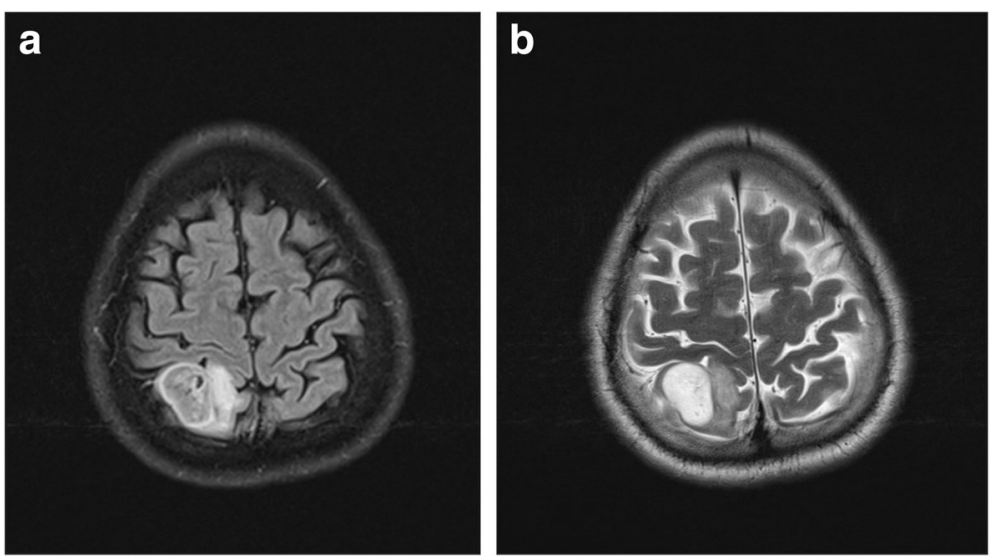

Fig. 2 a-b: a) FLAIR sequence demonstrating a relative hypointense signal with the exception of a hyperintense peripheral rim. b) T2W sequence demonstrating homogenous hyperintensive signal with a conspicuous border. This glioma was considered to have a mismatch sign and was diagnosed with an IDH-mutated and 1p19q codeleted glioma (i.e. oligodendroglioma)

\section{Interrater agreement}

We evaluated 215 cases for the mismatch sign and it was found in 17 cases (7.9\%), absent in 189 cases (87.9\%), while 9 cases were discordant (4.2\%), as demonstrated in Fig. 5. The total number of patients with mismatch sign was 21 (9.8\%) after we reached consensus for the 9 discordant cases (discordant cases are presented in Supplementary material). The interrater agreement for the mismatch sign between clinical neurosurgeons was at a kappa value of $0.74(p=0.064)$, and between clinical neurosurgeons and neuroradiologist $0.77(p<0.001)$.

\section{Molecular markers and genome-wide DNA methylation array, retrospective cohort}

DNA methylation profiling was performed for $I D H$-mut astrocytomas in the retrospective cohort where sufficient tumor tissue was available $(N=29)$ to determine of patients with a mismatch sign $(N=6)$ clustered together indicating a particular biological profile. Unsupervised hierarchical clustering with respect to the 5000 most deviating $\mathrm{CpG}$ sites in the methylation array grouped the $I D H$-mut astrocytomas into two main clusters as demonstrated in Fig. 6. However, patients with mismatch sign did not cluster together.

\section{Discussion}

In this study there were no differences between $I D H$ mut astrocytomas with or without the mismatch sign with respect to extent of resection or any other clinical outcome parameter. Further, $I D H$-mut non-codel gliomas (astrocytomas) with mismatch sign did not harbor a unique methylation profile. The only difference we found between the patients with $I D H$-mut astrocytomas with mismatch sign and those without was related to the imaging finding itself. However, we validate that the T2FLAIR mismatch sign has substantial interrater agreement and high specificity for $I D H$-mut astrocytomas.
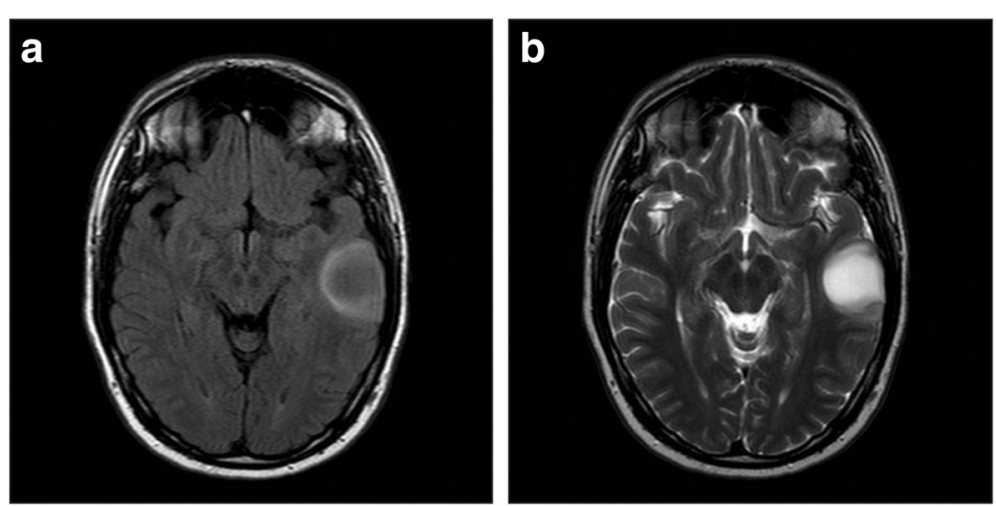

Fig. 3 a-b: a) FLAIR sequence demonstrating a relative hypointense signal with the exception of a hyperintense peripheral rim. b) T2W sequence demonstrating homogenous hyperintensive signal with a conspicuous border. This glioma was considered to have a mismatch sign and was diagnosed with an IDH-mutated and 1p19q codeleted glioma (i.e. oligodendroglioma) 

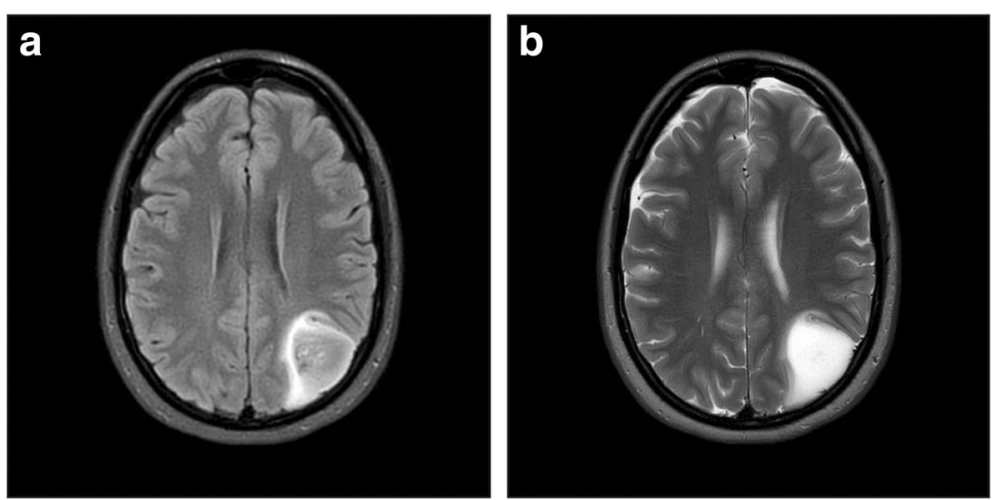

Fig. 4 a-b: a) FLAIR sequence demonstrating a relative hypointense signal with the exception of a hyperintense peripheral rim. b) T2W sequence demonstrating homogenous hyperintensive signal with a conspicuous border. This glioma was considered to have a mismatch sign and the histopathological diagnosis was glioblastoma (i.e. astrocytoma WHO grade IV, IDH-mut)

The clinical implications of mismatch sign are currently limited to this particular association.

During surgery it is evident that gliomas may vary in texture and appearance, and with the radiological image of homogenous signal on T2-weighted sequences and seemingly conspicuous tumor border, the question was raised whether the extent of the resection is related to the mismatch sign. This may be of particular importance, since the $I D H$-mut astrocytoma group seems to be the one benefitting most from extensive surgery $[13,14$, 26]. In our cohort, the extent of resection did not differ between groups, hence mismatch sign should not be taken as a factor influencing extent of resection in $\mathrm{IDH}$ mut astrocytomas. To our knowledge, this is the first study investigating the clinical factors and extent of resection in relation to the mismatch sign. One previous study evaluated the association between survival and the mismatch sign, with a median follow-up of 65.7 months, and found no differences in overall survival between groups [7]. This finding that mismatch sign does not indicate a particular prognostic group is further corroborated by our data.

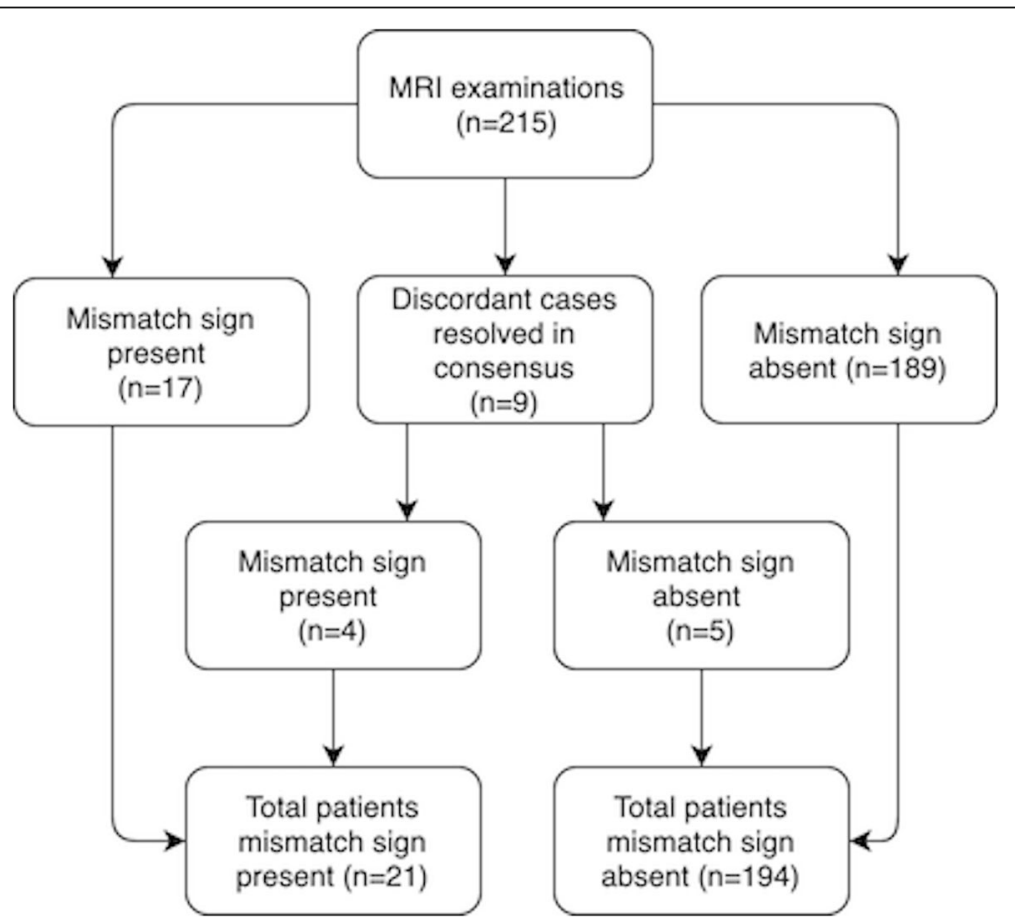

Fig. 5 Overview over MRI mismatch sign assessment and agreement among raters 


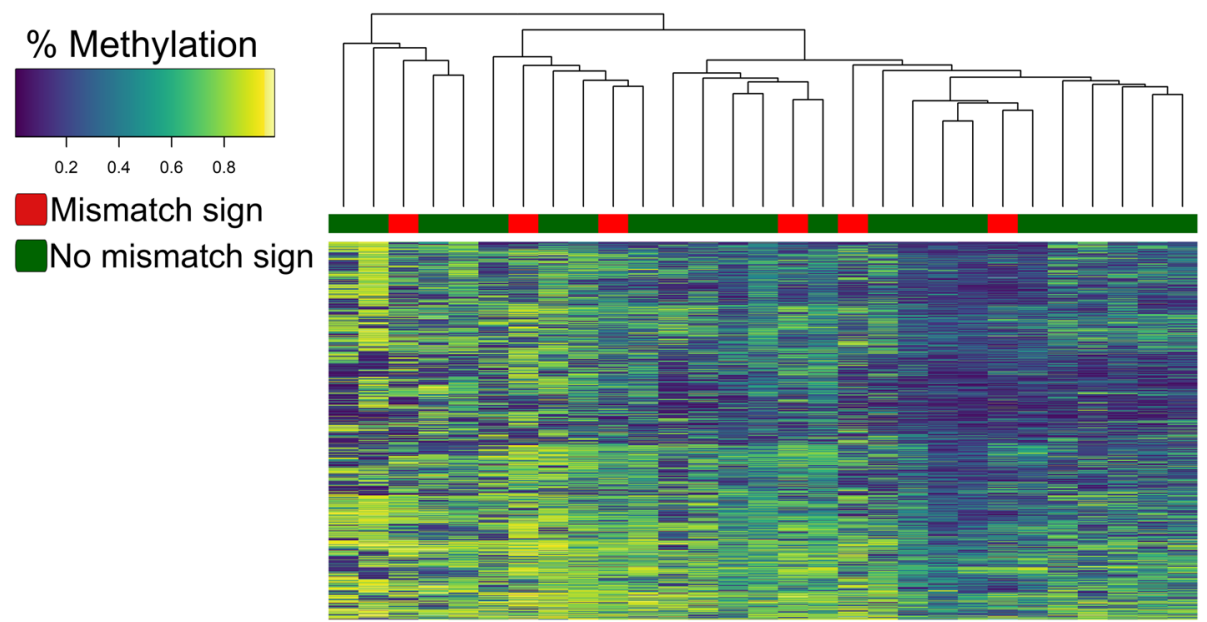

Fig. 6 Unsupervised hierarchical clustering analysis on methylation levels for the 5000 most deviating CpG sites in the EPIC methylation array. A value of 0.2 indicated low percentage of methylation and 0,8 a high percentage of methylation. Clustering of the DNA-methylation profiles for the IDH-mut astrocytomas in the retrospective cohort $(\mathrm{N}=29)$ did not identify a T2-FLAIR mismatch-methylation associated phenotype

Our results on interrater variability validate the data from Broen et al, who found a kappa value of 0.75 , which is considered a substantial interrater agreement $[17,25]$. According to the literature, a kappa value within the range of $0.56-0.79$ is moderate to substantial $[7,17,25,27]$. Thus, the collective experience so far is that the T2-FLAIR mismatch sign can reliably be detected in clinical practice $[7,17,27]$.

It should be noted that we tested the diagnostic properties of the mismatch sign with different patient selections, which is a strength compared to a pure histopathological selection in previous studies [7, 12, 17]. In our selection based upon histopathology, we identified two patients with mismatch sign who had IDH-mut codel glioma, unlike previous studies by Broen et al and Patel et al who presented a $100 \%$ specificity for $I D H$-mut astrocytomas [7, 17]. However, later reports had made similar findings to ours, and the overall specificity reported in the literature is therefore in the range of $96.0-100.0 \%[7,8,12,17,28]$. The mismatch sign has been found occasionally in $I D H$-mut codel gliomas, but also in pediatric low-grade brain tumors. This far, the mismatch sign has been reported in pilomyxoid astrocytoma, LGG harboring MYB rearrangement, oligodendroglioma (IDH-mut codel), and even in one patient with a non-neoplastic lesion [8].

In our selection of patients with radiologically suspected LGG other tumor diagnoses may also be encountered. Indeed, one patient in this prospective cohort with T2-FLAIR mismatch sign had an $I D H$-mut glioblastoma, suggesting that the mismatch sign is not grade specific. Importantly, there were no other differential diagnoses beyond diffuse gliomas that presented with the mismatch sign. Although of low sensitivity (27.1-51.0\%), the specificity for $I D H$-mut astrocytomas renders the evaluation of mismatch sign useful in a clinical setting for individual cases [12, 17, 28, 29]. Adding advanced imaging parameters like apparent diffusion coefficient (ADC) and cerebral blood volume (CBV) to the mismatch sign may further improve the diagnostic capabilities of $I D H$-mut astrocytomas, although at the cost of increased complexity [30-33].

In an effort to understand the biological importance of the mismatch sign, we used DNA methylation analyses and unsupervised hierarchical clustering in a small subsample of patients with $I D H$-mut astrocytoma from the retrospective cohort. Clustering analysis could not distinguish between samples with mismatch sign from those without. Thus, this could indicate that the mismatch sign did not have a common overall methylation profile. The only other in-depth analysis of biology so far was performed by Patel el al, who found no convincing differences in biology, including methylation analysis [34]. Finally, since survival is consistently reported not to differ between groups with or without mismatch sign [7], it seems unlikely that the patients with mismatch sign constitute a specific type of $I D H$-mut astrocytomas.

\section{Strength and limitations}

Strengths of this study include the both histopathological and image-based selection in the evaluation of the T2-FLAIR mismatch sign. Our prospective cohort of patients with suspected LGG reflects clinical neurooncology practice, where also other relevant diagnoses may be encountered at times. The small sample size is one limitation, especially for the methylation subsample analyses. For survival analyses, a longer follow-up would have been preferable, as a part of the cohort recently underwent surgical treatment. Since this was an 
exploratory study of clinical factors associated with the mismatch sign, we did not adjust for multiple comparisons and thereby increasing the chance of false positive associations simply by chance. However, we did not find any significant association, even without this adjustment.

\section{Conclusion}

The T2-FLAIR mismatch sign in patients with $I D H$-mut astrocytomas was not found to be associated with clinical variables such as presenting symptoms, extent of resection, or survival. Methylation analysis further strengthens the previous indications that the $I D H$-mut astrocytomas with mismatch sign does not compromise a specific subentity. Finally, we validate the T2-FLAIR mismatch sign as a reliable marker with high specificity of $I D H$-mut astrocytomas, but with limited sensitivity.

\section{Supplementary information}

Supplementary information accompanies this paper at https://doi.org/10. 1186/s12885-020-06951-w.

Additional file 1: Supplementary material. "Data discordant cases T2FLAIR mismatch sign"

Additional file 2: Supplementary Fig. 1a-b. I a) FLAIR sequence demonstrating a relative hypointense signal with the exception of a hyperintense peripheral rim. b) T2W sequence demonstrating homogenous hyperintensive signal with a conspicuous border. This glioma was considered to have a mismatch sign.

Additional file 3: Supplementary Fig. 2a-b. a) FLAIR sequence demonstrating a relative hypointense signal with the exception of a hyperintense peripheral rim. b) T2W sequence demonstrating homogenous hyperintensive signal with a conspicuous border. This glioma was considered to have a mismatch sign.

Additional file 4: Supplementary Fig. 3a-b. a) FLAIR sequence demonstrating hyperintensive signal with diffuse border. b) T2W sequence demonstrating hyperintensive signal with diffuse border. This glioma was considered not to have a mismatch sign.

\section{Abbreviations}

ADC: Apparent diffusion coefficient; CBV: Cerebral blood volume; Codel: 1p19q codeleted; DNET: Dysembryoplastic neuroepithelial tumor; FLAIR: Fluid-attenuated inversion recovery; IDH1 and 2: Isocitrate dehydrogenase genes 1 and 2; IDH-mut: Isocitrate dehydrogenase gene mutation; LGG: Lower-grade gliomas, defined as WHO grade II and grade III diffuse gliomas; MDT: Multidisciplinary team; MRI: Magnetic resonance imaging; NPV: Negative predictive value; Non-codel: 1p19q non-codeleted; NPV: Negative predictive value; Mismatch sign: T2-FLAIR mismatch sign

\section{Acknowledgments}

We would like to thank Tomás Gómez Vecchio, research assistant at the institute of Neuroscience and Physiology, Sahlgrenska Academy, Gothenburg, Sweden, for assistance with data management.

\section{Authors' contributions}

AC, AS, LC, ASJ, NH and IBB performed the clinical and radiological data collection and analysis. SFV, HC and TOB performed laboratory analysis and collected tissue for analysis. AC performed the statistical analyses. AC, AS and AJ drafted the manuscript. All authors substantively revised the manuscript and have approved the submitted version.

\section{Funding}

This study received financial support in the form of ALF-grant (ALFGBG716671 and ALFGBG2018-03591), funds of the Sahlgrenska University
Hospital and funding from the Swedish Research Council (2017-00944). The sponsors had no role in the design or conduct of this research.

Open access funding provided by University of Gothenburg.

\section{Availability of data and materials}

The datasets used and analyzed during the current study are available from the corresponding author on reasonable request.

\section{Ethics approval and consent to participate}

This project was approved by the regional ethical committee in the region of Västra Götaland (DNR 1067-16 and DNR 363-17).

\section{Consent for publication}

Not applicable. Informed consent waived by the ethical committee.

\section{Competing interests}

All authors certify that they have no affiliations with or involvement in any organization or entity with any financial interest (such as honoraria; educational grants; participation in speakers' bureaus; membership, employment, consultancies, stock ownership, or other equity interest; and expert testimony or patent-licensing arrangements), or non-financial interest (such as personal or professional relationships, affiliations, knowledge or beliefs) in the subject matter or materials discussed in this manuscript.

\section{Author details}

'Department of Neurosurgery, Sahlgrenska University Hospital, Gothenburg, Sweden. ${ }^{2}$ Department of Clinical Neuroscience, Institute of Neuroscience and Physiology, University of Gothenburg, Sahlgrenska Academy, Gothenburg, Sweden. ${ }^{3}$ Department of Radiology, Sahlgrenska University Hospital, Gothenburg, Sweden. ${ }^{4}$ Department of Radiology, Institute of Clinical Sciences, Sahlgrenska Academy, University of Gothenburg, Gothenburg, Sweden. ${ }^{5}$ Department of Neuroscience, Neurology, Uppsala University, Uppsala, Sweden. ${ }^{6}$ Department of Clinical Pathology and Cytology, Sahlgrenska University Hospital, Gothenburg, Sweden. 'Department of Physiology, Institute of Neuroscience and Physiology, University of Gothenburg, Sahlgrenska Academy, Gothenburg, Sweden. ${ }^{8}$ Sahlgrenska Cancer Center, Department of Laboratory Medicine, Institute of Biomedicine, Sahlgrenska Academy, University of Gothenburg, Gothenburg, Sweden. ${ }^{9}$ Department of Neuromedicine and Movement Science, NTNU, Trondheim, Norway.

Received: 12 December 2019 Accepted: 11 May 2020 Published online: 20 May 2020

\section{References}

1. Louis DN, Perry A, Reifenberger G, von Deimling A, Figarella-Branger D, Cavenee WK, Ohgaki H, Wiestler OD, Kleihues P, Ellison DW. The 2016 World Health Organization classification of tumors of the central nervous system: a summary. Acta Neuropathol. 2016;131(6):803-20.

2. Brat DJ, Verhaak RG, Aldape KD, Yung WK, Salama SR, Cooper LA, Rheinbay E, Miller CR, Vitucci M, Morozova O, et al. Comprehensive, integrative genomic analysis of diffuse lower-grade Gliomas. N Engl J Med. 2015; 372(26):2481-98.

3. Claus EB, Walsh KM, Wiencke JK, Molinaro AM, Wiemels JL, Schildkraut JM, Bondy ML, Berger M, Jenkins R, Wrensch M. Survival and low-grade glioma: the emergence of genetic information. Neurosurg Focus. 2015;38(1):E6.

4. Etxaniz O, Carrato C, de Aguirre I, Queralt C, Munoz A, Ramirez JL, Rosell R, Villa S, Diaz R, Estival A, et al. IDH mutation status trumps the Pignatti risk score as a prognostic marker in low-grade gliomas. J Neuro-Oncol. 2017; 135(2):273-84

5. Sun H, Yin L, Li S, Han S, Song G, Liu N, Yan C. Prognostic significance of IDH mutation in adult low-grade gliomas: a meta-analysis. J Neuro-Oncol. 2013;113(2):277-84.

6. Eckel-Passow JE, Lachance DH, Molinaro AM, Walsh KM, Decker PA, Sicotte H, Pekmezci M, Rice T, Kosel ML, Smirnov IV, et al. Glioma groups based on 1p/19q, IDH, and TERT promoter mutations in tumors. N Engl J Med. 2015; 372(26):2499-508.

7. Patel SH, Poisson LM, Brat DJ, Zhou Y, Cooper L, Snuderl M, Thomas C, Franceschi AM, Griffith B, Flanders AE, et al. T2-FLAIR mismatch, an imaging biomarker for IDH and 1p/19q status in lower-grade Gliomas: a TCGA/TCIA project. Clin Cancer Res. 2017;23(20):6078-85. 
8. Johnson DR, Kaufmann TJ, Patel SH, Chi AS, Snuderl M, Jain R. There is an exception to every rule-T2-FLAIR mismatch sign in gliomas. Neuroradiology. 2019;61(2):225-7.

9. Capper D, Jones DTW, Sill M, Hovestadt V, Schrimpf D, Sturm D, Koelsche C, Sahm F, Chavez L, Reuss DE, et al. DNA methylation-based classification of central nervous system tumours. Nature. 2018:555(7697):469-74.

10. Wiestler B, Capper D, Sill M, Jones DT, Hovestadt V, Sturm D, Koelsche C, Bertoni A, Schweizer L, Korshunov A, et al. Integrated DNA methylation and copy-number profiling identify three clinically and biologically relevant groups of anaplastic glioma. Acta Neuropathol. 2014;128(4):561-71.

11. Christensen BC, Smith AA, Zheng S, Koestler DC, Houseman EA, Marsit CJ, Wiemels JL, Nelson HH, Karagas MR, Wrensch MR, et al. DNA methylation, isocitrate dehydrogenase mutation, and survival in glioma. J Natl Cancer Inst. 2011;103(2):143-53.

12. Juratli TA, Tummala SS, Riedl A, Daubner D, Hennig S, Penson T, Zolal A, Thiede C, Schackert G, Krex D, et al. Radiographic assessment of contrast enhancement and T2/FLAIR mismatch sign in lower grade gliomas: correlation with molecular groups. J Neuro-Oncol. 2019;141(2):327-35.

13. Delev D, Heiland DH, Franco P, Reinacher P, Mader I, Staszewski O, Lassmann S, Grau S, Schnell O. Surgical management of lower-grade glioma in the spotlight of the 2016 WHO classification system. J Neuro-Oncol. 2019; 141(1):223-33.

14. Wijnenga MMJ, French PJ, Dubbink HJ, Dinjens WNM, Atmodimedjo PN, Kros JM, Smits M, Gahrmann R, Rutten GJ, Verheul JB, et al. The impact of surgery in molecularly defined low-grade glioma: an integrated clinical, radiological, and molecular analysis. Neuro-oncology. 2018;20(1):103-12.

15. Ferroli P, Broggi M, Schiavolin S, Acerbi F, Bettamio V, Caldiroli D, Cusin A, La Corte E, Leonardi M, Raggi A, et al. Predicting functional impairment in brain tumor surgery: the big five and the Milan complexity scale. Neurosurg Focus. 2015;39(6):E14.

16. Louis DN, Ohgaki H, Wiestler OD, Cavenee WK, Burger PC, Jouvet A, Scheithauer BW, Kleihues P. The 2007 WHO classification of tumours of the central nervous system. Acta Neuropathol. 2007;114(2):97-109.

17. Broen MPG, Smits M, Wijnenga MMJ, Dubbink HJ, Anten M, Schijns O, Beckervordersandforth J, Postma AA, van den Bent MJ. The T2-FLAIR mismatch sign as an imaging marker for non-enhancing IDH-mutant, 1p/19q-intact lower-grade glioma: a validation study. Neuro-Oncology. 2018;20(10):1393-9.

18. Mor V, Laliberte L, Morris JN, Wiemann M. The Karnofsky performance status scale. An examination of its reliability and validity in a research setting. Cancer. 1984;53(9):2002-7.

19. Chang EF, Smith JS, Chang SM, Lamborn KR, Prados MD, Butowski N, Barbaro NM, Parsa AT, Berger MS, McDermott MM. Preoperative prognostic classification system for hemispheric low-grade gliomas in adults. J Neurosurg. 2008;109(5):817-24.

20. Fedorov A, Beichel R, Kalpathy-Cramer J, Finet J, Fillion-Robin JC, Pujol S, Bauer C, Jennings D, Fennessy F, Sonka M, et al. 3D slicer as an image computing platform for the quantitative imaging network. Magn Reson Imaging. 2012;30(9):1323-41.

21. Wenger A, Ferreyra Vega S, Kling T, Bontell TO, Jakola AS, Caren H Intratumor DNA methylation heterogeneity in glioblastoma: implications for DNA methylation-based classification. Neuro-Oncol. 2019;21(5):616-27.

22. Aryee MJ, Jaffe AE, Corrada-Bravo H, Ladd-Acosta C, Feinberg AP, Hansen KD, Irizarry RA. Minfi: a flexible and comprehensive bioconductor package for the analysis of Infinium DNA methylation microarrays. Bioinformatics. 2014;30(10):1363-9.

23. Triche TJ Jr, Weisenberger DJ, Van Den Berg D, Laird PW, Siegmund KD. Low-level processing of Illumina Infinium DNA methylation BeadArrays. Nucleic Acids Res. 2013;41(7):e90.

24. Fortin JP, Triche TJ Jr, Hansen KD. Preprocessing, normalization and integration of the Illumina HumanMethylationEPIC array with minfi. Bioinformatics. 2017;33(4):558-60.

25. Landis JR, Koch GG. The measurement of observer agreement for categorical data. Biometrics. 1977;33(1):159-74

26. Jakola AS, Skjulsvik AJ, Myrmel KS, et al. Surgical resection versus watchful waiting in low-grade gliomas. Ann Oncol. 2017;28(8):1942-48.

27. Batchala PP, Muttikkal TJE, Donahue JH, Patrie JT, Schiff D, Fadul CE, Mrachek EK, Lopes MB, Jain R, Patel SH. Neuroimaging-based classification algorithm for predicting 1p/19q-Codeletion status in IDH-mutant lower grade Gliomas. AJNR Am J Neuroradiol. 2019;40(3):426-32.

28. Lasocki A, Gaillard F, Gorelik A, Gonzales M. MRI features can predict 1p/19q status in intracranial Gliomas. AJNR Am J Neuroradiol. 2018;39(4):687-92.
29. Ranganathan P, Aggarwal R. Common pitfalls in statistical analysis: understanding the properties of diagnostic tests - part 1. Perspect Clin Res. 2018;9(1):40-3.

30. Cho HH, Lee $\mathrm{SH}$, Kim J, Park H. Classification of the glioma grading using radiomics analysis. PeerJ. 2018;6:e5982.

31. Gui C, Lau JC, Kosteniuk SE, Lee DH, Megyesi JF. Radiology reporting of lowgrade glioma growth underestimates tumor expansion. Acta Neurochir. 2019;161(3):569-76.

32. Jakola AS, Reinertsen I. Radiological evaluation of low-grade glioma: time to embrace quantitative data? Acta Neurochir. 2019;161(3):577-8.

33. Lee MK, Park JE, Jo Y, Park SY, Kim SJ, Kim HS. Advanced imaging parameters improve the prediction of diffuse lower-grade gliomas subtype, IDH mutant with no 1p19q codeletion: added value to the T2/FLAIR mismatch sign. Eur Radiol. 2020;30(2):844-54.

34. de Souza CF, Sabedot TS, Malta TM, Stetson L, Morozova O, Sokolov A, Laird PW, Wiznerowicz M, lavarone A, Snyder J, et al. A distinct DNA methylation shift in a subset of Glioma CpG Island Methylator phenotypes during tumor recurrence. Cell Rep. 2018;23(2):637-51.

\section{Publisher's Note}

Springer Nature remains neutral with regard to jurisdictional claims in published maps and institutional affiliations.
Ready to submit your research? Choose BMC and benefit from:

- fast, convenient online submission

- thorough peer review by experienced researchers in your field

- rapid publication on acceptance

- support for research data, including large and complex data types

- gold Open Access which fosters wider collaboration and increased citations

- maximum visibility for your research: over $100 \mathrm{M}$ website views per year

At BMC, research is always in progress.

Learn more biomedcentral.com/submissions 